\title{
Myelin-associated glycoprotein gene and brain morphometry in schizophrenia
}

\section{Daniel Felsky ${ }^{1}$, Aristotle N. Voineskos ${ }^{1}$, Jason P. Lerch ${ }^{2}$, Arash Nazeri ${ }^{1,3}$, Sajid A. Shaikh ${ }^{1}$, Tarek K. Rajji ${ }^{1}$, Benoit H. Mulsant ${ }^{1}$ and James L. Kennedy ${ }^{1}{ }^{*}$}

\author{
${ }_{1}^{1}$ Neuroscience Research Department, Centre for Addiction and Mental Health, University of Toronto, Toronto, ON, Canada \\ ${ }^{2}$ Department of Neurosciences and Mental Health, Hospital for Sick Children, University of Toronto, Toronto, ON, Canada \\ ${ }^{3}$ Interdisciplinary Neuroscience Research Program, Tehran University of Medical Sciences, Tehran, Iran
}

\section{Edited by:}

Martin Lepage, Douglas Mental

Health University Institute, Canada

Reviewed by:

David Luck, Université de Montréal, Canada

Neeltje E. M. Van Haren, University

Medical Center Utrecht, Netherlands

\section{*Correspondence:}

James L. Kennedy, Neuroscience Research Department, Centre for Addiction and Mental Health, 250 College St, Toronto, ON, Canada M5T 1 R8.

e-mail: james_kennedy@camh.net
Myelin and oligodendrocyte disruption may be a core feature of schizophrenia pathophysiology. The purpose of the present study was to localize the effects of previously identified risk variants in the myelin-associated glycoprotein $(M A G)$ gene on brain morphometry in schizophrenia patients and healthy controls. Forty-five schizophrenia patients and 47 matched healthy controls underwent clinical, structural magnetic resonance imaging, and genetics procedures. Gray and white matter cortical lobe volumes along with hippocampal volumes were calculated from T1-weighted MRI scans. Each subject was also genotyped for the two disease-associated MAG single nucleotide polymorphisms (rs720308 and rs720309). Repeated measures general linear model (GLM) analysis found significant region by genotype and region by genotype by diagnosis interactions for the effects of MAG risk variants on lobar gray matter volumes. No significant associations were found with lobar white matter volumes or hippocampal volumes. Follow-up univariate GLMs found the AA genotype of rs720308 predisposed schizophrenia patients to left temporal and parietal gray matter volume deficits. These results suggest that the effects of the MAG gene on cortical gray matter volume in schizophrenia patients can be localized to temporal and parietal cortices. Our results support a role for MAG gene variation in brain morphometry in schizophrenia, align with other lines of evidence implicating MAG in schizophrenia, and provide genetically based insight into the heterogeneity of brain imaging findings in this disorder.

Keywords: schizophrenia, myelin-associated glycoprotein, imaging-genetics, temporal lobe, parietal lobe, gray matter volume

\section{INTRODUCTION}

Changes in myelin- and oligodendrocyte-related genes have been implicated in the pathophysiology of schizophrenia. Postmortem studies of multiple brain regions show dysregulation of myelinassociated genes in schizophrenia (Fannon et al., 2000; Hakak et al., 2001; Aston et al., 2004; Katsel et al., 2005; Haroutunian et al., 2007) and of these, the myelin-associated glycoprotein (MAG) gene is consistently among the most down-regulated. Additionally, two candidate gene studies have found associations of $M A G$ variants with schizophrenia: Yang et al. (2005), in a transmission disequilibrium test (TDT) analysis of 413 Chinese Han trios, found positive associations with two-marker haplotypes of rs720308 and rs720309, and Wan et al. (2005), using a case-control design $\left(n_{\text {case }}=470, n_{\text {control }}=470\right.$, also Chinese Han $)$, found the same markers significantly associated with schizophrenia. More recently, we found a significant association between $M A G$ genotype at these same loci and total cortical gray matter volume in first episode schizophrenia patients (Voineskos et al., 2008), however, no study has yet examined the regional effects of these SNPs on the brain.

Brain matter abnormalities in the frontal and temporal cortices are among the most common findings in neuroimaging studies of schizophrenia (Fornito et al., 2009; Shenton et al., 2010).
Structural analyses of brain changes in schizophrenia have principally found volume deficits in the superior temporal gyrus as well as medial temporal lobe structures, such as the hippocampus and parahippocampal gyrus (Shenton et al., 2010). Abnormalities of the prefrontal cortex have also been identified in schizophrenia (Shenton et al., 2010). Disruptions of the parietal and occipital lobes, though less studied than other brain regions in schizophrenia, may also be features of this disease. Schlaepfer et al. (1994) and Ross and Pearlson (1996) have demonstrated reductions in gray matter of the heteromodal association cortex, which includes the inferior parietal lobule, in schizophrenia patients.

Biologically convergent lines of evidence have independently implicated $M A G$ locus variation and cortical gray matter volume deficits as potential schizophrenia liability factors. The present study builds on our previous association of $M A G$ with total cortical gray matter volume in schizophrenia by analyzing the effect of $M A G$ genotype on regional cortical brain gray and white matter volumes. We also examined effects of genotype on the hippocampus since there is evidence for altered $M A G$ regulation in schizophrenia in this region (Dracheva et al., 2006). We hypothesized, based on postmortem data and our previous work, that MAG genotype would predict variation in cortical gray matter volumes, 
particularly in frontal and temporal brain regions. Furthermore, given altered expression of the $M A G$ gene in schizophrenia, as well as our previous findings of reduced cortical gray mater in schizophrenia patients only, we hypothesized that there may be a genotype by diagnosis interaction predicting variation in brain imaging measures.

\section{MATERIALS AND METHODS SAMPLE SELECTION}

Study participants were recruited at the Centre for Addiction and Mental Health (CAMH) in Toronto, Canada by referral, study registries, and advertisements. To characterize clinical symptoms, participants were administered the structured clinical interview for DSM-IV disorders (SCID; First et al., 1995) and the positive and negative syndrome scale (PANSS), and were interviewed by a psychiatrist at CAMH to ensure diagnostic accuracy. Details on history of medication were gathered by self-report and verified when necessary by the treating psychiatrist and chart review. All participants were Caucasian and between 18 and 59 years of age. Exclusion criteria for patients included any history of substance dependence or abuse within the past 6 months, previous head trauma, loss of consciousness, and neurological disorders. For controls, additional exclusion criteria included any history of a primary psychotic disorder in first-degree relatives. Controls were group matched to patients based on age, gender, and handedness (Edinburgh handedness inventory; Oldfield, 1971). Written and informed consent was obtained and the study was approved by the CAMH Ethics Review Board.

\section{GENOTYPING}

Subjects were genotyped for two SNPs at the MAG locus (rs720308 and rs720309). DNA was extracted from blood samples using a modified high-salt method (Lahiri and Nurnberger, 1991), and checked for concentration using the NanoDrop 8000 spectrophotometer. Samples were deemed acceptable if their concentration was $>0.02 \mu \mathrm{g} / \mu \mathrm{l}$ and optical density (OD) A260/280 ratio between 1.5 and 2.5. Samples were then diluted to a final concentration of $20 \mathrm{ng} / \mu \mathrm{l}$ for genotype analysis. Genotyping was done using the Taqman Assay-on-Demand protocol with a total PCR reaction volume of $10 \mu \mathrm{l}$. Markers were analyzed post-amplification using the Applied Biosystems 7500 Real-Time PCR System for allelic discrimination, and calls were made manually. Two lab personnel independently verified results and $10 \%$ of sample genotypes were duplicated for quality control.

\section{IMAGE ACOUISITION}

High resolution magnetic resonance images were acquired as part of a multi-modal imaging protocol using an eight-channel head coil on a 1.5-T GE Echospeed system (General Electric Medical Systems, Milwaukee, WI, USA), which permits maximum gradient amplitudes of $40 \mathrm{mT} / \mathrm{m}$. Axial inversion recovery prepared spoiled gradient recall images were acquired: echo time $(\mathrm{TE})=5.3 \mathrm{~ms}$, repetition time $(\mathrm{TR})=12.3 \mathrm{~ms}$, time to inversion $(\mathrm{TI})=300 \mathrm{~ms}$, flip angle $=20^{\circ}$, number of excitations $(\mathrm{NEX})=1$ (124 contiguous images, $1.5 \mathrm{~mm}$ thickness).

\section{IMAGE PROCESSING}

For cortical volume analysis, each subject's T1 image was submitted to the CIVET pipeline (version 1.1.7 developed at the
Montreal Neurologic Institute; Ad-Dab'bagh et al., 2006). The processing steps included registration to the symmetric ICBM 152 template (Mazziotta et al., 2001) with a 12-parameter linear transformation (Collins, 1994), correction for inhomogeneity artifact (Sled et al., 1998), skull stripping (Smith et al., 2002), tissue classification into white and gray matter, cerebrospinal fluid and background (Zijdenbos et al., 2002; Tohka et al., 2004), and neuroanatomical segmentation using ANIMAL (Collins et al., 1995). Total volumes for each cortical lobe were estimated for each individual by non-linearly warping each $\mathrm{T} 1$ image toward a segmented atlas. Volume $\left(\mathrm{mm}^{3}\right)$ was extracted from each of these regions using the RMINC package (version 0.4) for reading and analyzing MINC2 output files. Total gray matter, white matter, and CSF volumes were calculated, along with lobar cortical gray and white matter volumes (i.e., left and right frontal, temporal, parietal, occipital).

For hippocampal volumes, each individual's T1-weighted image was processed using the FMRIB's Integrated Registration and Segmentation Tool (FIRST v1.2) automated subcortical segmentation pipeline (Patenaude, 2007; Patenaude et al., 2011) included in the open access FSL (v.4.1.8) package. This method uses a Bayesian modeling framework based on multivariate Gaussian assumptions to estimate the shape and appearance of subcortical structures. To achieve this, it employs point distribution and surface mesh models generated from a set of 336 manually segmented images (provided by the Centre for Morphometric Analysis (CMA), MGH, Boston). Each subject's image was registered using FLIRT (Jenkinson et al., 2002) in a two-step transformation (each step using 12 degrees of freedom) that first affine-registered whole-head images to non-linear MNI152 space ( $1 \mathrm{~mm}$ resolution) and then excluded voxels outside a subcortical mask (generated from the filled average shapes of all subcortical structures being segmented in the MNI space). The automated segmentation process then computed the most probable shape of each structure by comparing linear combinations of shape principal components from the manually segmented models to the observed intensities from each input image. The resulting vertex models were then transformed back to native space, boundaries corrected, and volumes were calculated for left and right hippocampus.

Brain tissue volume, normalized for subject head size, was estimated with SIENAX (Smith et al., 2001; Smith, 2002), also part of the FSL toolkit. First, brain and skull images were extracted from the single whole-head images (Smith, 2002) and then affineregistered to MNI152 space (Jenkinson and Smith, 2001) (using the skull image to determine registration scaling). Next, tissuetype segmentation with partial volume estimation was carried out (Zhang et al., 2001) in order to calculate total volume of brain tissue (including separate volume estimates of gray matter, white matter, peripheral gray matter, and ventricular CSF).

\section{STATISTICAL ANALYSIS}

Statistical packages for the social sciences (SPSS) version 19 was used for statistical analysis. Individuals were grouped by genotype at rs720308 and rs720309 as either major allele (A/T) homozygotes or minor allele (G/A) carriers and a repeated measures general linear model (GLM) was applied. Separate analyses were carried out for lobar gray and white matter measures. For each cortical analysis, genotype and diagnosis were between-group factors, and eight 
within-group factors corresponding to left and right frontal, temporal, parietal, and occipital lobe volumes were used, with age and total brain volume as covariates. For hippocampal volume, a separate repeated measures analysis with two within-group factors (left and right hippocampus) was applied to determine effects of diagnosis and genotype, with total brain volume and age as covariates. Where repeated measures analyses showed significant associations, post hoc univariate GLM analysis was used to localize effects of genotype and/or diagnosis on brain morphometry measures.

\section{RESULTS}

A 100\% successful genotyping rate was achieved. MAG genotype distribution was not significantly different between schizophrenia patients and controls and did not violate Hardy-Weinberg equilibrium $(p=0.578)$. The SNPs under investigation were in full linkage disequilibrium $\left(D^{\prime}=1\right)$ in this sample. Therefore, we proceeded with imaging-genetics analysis using only one of the two SNPs (we chose rs720308). There were no significant differences in gender $(p=0.503)$, age $(p=0.137)$, or ethnicity (all Caucasian) between the group of schizophrenia patients $(n=45)$ and controls $(n=47$; Table 1).

Repeated measures GLM found a significant region by genotype interaction $\left(F_{7,602}=2.8, p=0.007\right.$, Greenhouse-Geisser correction: $\left.F_{4,317}=2.8, p=0.031\right)$ and significant region by genotype by diagnosis interaction $\left(F_{7,602}=3.2, p=0.003\right.$, GreenhouseGeisser correction: $\left.F_{4,317}=3.2, p=0.016\right)$ for lobar gray matter volumes. No significant associations were found with lobar white matter volumes (Genotype by region: $F_{7,602}=0.45, p=0.87$; genotype by region by diagnosis: $F_{7,602}=1.6, p=0.12$ ) or hippocampal volumes (Genotype by region: $F_{1,86}=0.21, p=0.65$; genotype by region by diagnosis: $F_{1,86}=2.43, p=0.12$ ).

Follow-up univariate GLM on bilateral lobar gray matter volumes found a main effect of diagnosis for frontal lobe volume $\left(F_{5,86}=5.361, p=0.023\right)$, a main effect of genotype for temporal lobe volume $\left(F_{5,86}=7.318, p=0.008\right)$, and a significant genotype by diagnosis interaction for parietal lobe volume $\left(F_{5,86}=6.478\right.$, $p=0.0127)$. However, after Bonferroni correction for four comparisons $(p=0.0125)$, only the temporal lobe finding remains significant, with the parietal lobe bordering on significance.

Further investigation of left and right hemispheres localized the temporal and parietal findings to the left side $(F=7.742$, $p=0.007$ and $F=7.930, p=0.006$, respectively), with analyses of the right temporal and parietal lobes failing to survive correction for multiple comparisons $(F=4.852, p=0.03$ and $F=3.245$, $p=0.075$, respectively). The AA genotype predisposed both schizophrenia patients and healthy controls to lower left temporal gray matter volume (Figure 1). In addition, this genotype predisposed schizophrenia patients to lower left parietal gray matter volume (Figure 2). Chlorpromazine (CPZ) equivalents of medication exposure ( $\mathrm{mg} /$ day) were calculated for the schizophrenia patients (mean $=361, \mathrm{SD}=318$ ) and regressed against lobar volumetric measures. No significant correlation of $\mathrm{CPZ}$ equivalents was found with any lobar brain measure.

\section{DISCUSSION}

The present study used a carefully matched sample of schizophrenia patients and healthy controls to examine the effects of putative $M A G$ gene risk variants on brain morphometry. Our main findings were that variation in the $M A G$ gene influenced temporal lobe gray matter volume in both schizophrenia patients and healthy controls, and left parietal lobe gray matter volume in schizophrenia patients. Our findings localize previously shown effects of the $M A G$ gene on cortical gray matter to temporal and parietal regions. No association of this locus with white matter volumes or hippocampus was found.

Myelin system genes may play a key role in cortical brain growth, development, and maintenance, as these genes are critical in oligodendrocyte growth, maintenance, and repair during the

Table 1 | Sample demographics information including $p$-value of group difference between controls and schizophrenics.

\begin{tabular}{|c|c|c|c|}
\hline Demographic & Controls $(n=47)$, mean (SD) & Schizophrenics $(n=45)$, mean (SD) & Difference $(p)$ \\
\hline Age & $38(13)$ & $42(14)$ & 0.137 \\
\hline Education & $16(2)$ & $13(3)$ & $<0.001$ \\
\hline MMSE & $29(1)$ & $29(1)$ & 0.144 \\
\hline CIRS-G & $0.6(0.6)$ & $2(0.6)$ & $<0.001$ \\
\hline Positive & NA & $14(5)$ & NA \\
\hline Negative & NA & $15(6)$ & NA \\
\hline General & NA & $26(7)$ & NA \\
\hline Total & NA & $55(16)$ & NA \\
\hline Gender & $13 \mathrm{~F}, 34 \mathrm{M}$ & $16 \mathrm{~F}, 29 \mathrm{M}$ & 0.503 \\
\hline
\end{tabular}

Age, Education, WTAR, MMSE, and CIRS-G differences were assessed using independent sample t-tests, and Gender differences were assessed using Fisher's exact test.

NA, not applicable; M, male; F, female; WTAR, Wechsler Test for Adult Reading; MMSE, Mini Mental State Examination; CIRS-G, Clinical Information Rating Scale, Geriatrics. 


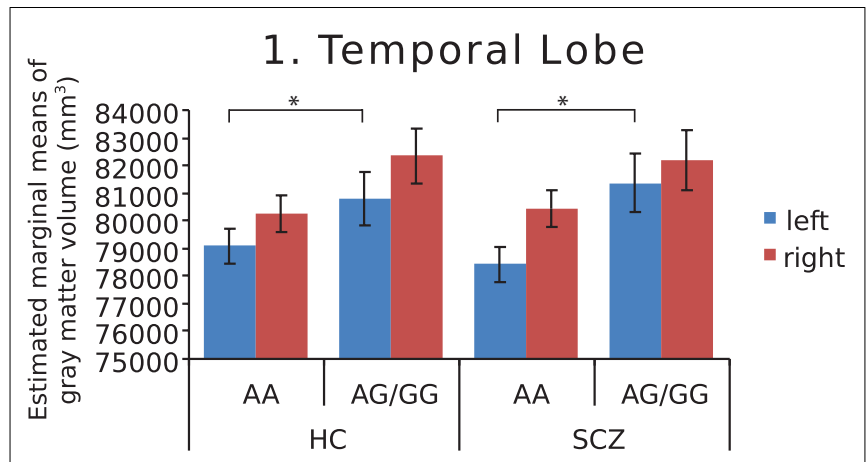

FIGURE 1 | Estimated marginal means of gray matter volume in the temporal lobe, by diagnosis and genotype at MAG rs720308, adjusted for age and total brain volume. Genotype effect $(F=7.742, p=0.007)$ is shown for left hemisphere. $(\mathrm{HC}$, healthy controls, SCZ, schizophrenic patients).

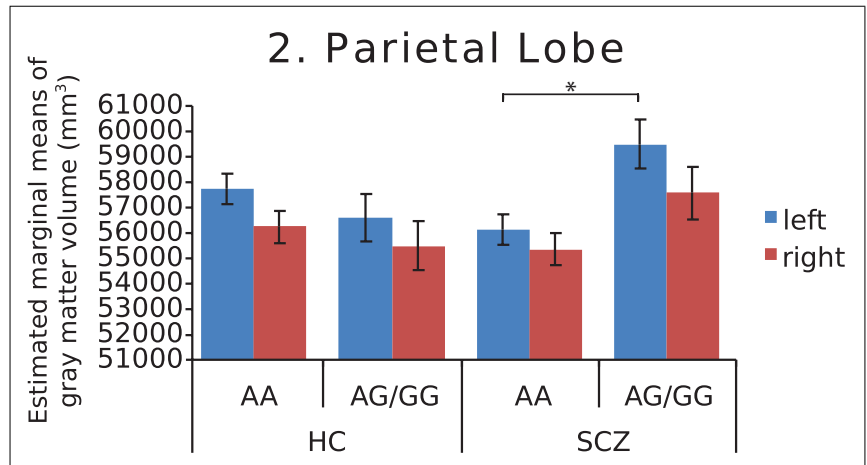

FIGURE 2 | Estimated marginal means of gray matter volume in the parietal lobe, by diagnosis and genotype at MAG rs720308, adjusted for age and total brain volume. Genotype by diagnosis interaction ( $F=7.930, p=0.006)$ is shown for left hemisphere. ( $\mathrm{HC}$, healthy controls, SCZ, schizophrenic patients).

adult lifespan. The $M A G$ gene specifically supports axon stability and impulse transmission through its maintenance of the intermembrane distance of the periaxonal space (Nguyen et al., 2009). Considerable evidence points to abnormalities in expression of the MAG gene in the temporal lobe in schizophrenia (Hakak et al., 2001; Katsel et al., 2005; Haroutunian et al., 2007), aligning with temporal lobe volume reductions in this disorder. Furthermore, temporal lobe volume deficits in the left hemisphere are reported more frequently than those in the right (Sun et al., 2009). Our finding of $M A G$ gene variation influence on left temporal lobe volume in healthy controls may represent an intermediate risk phenotype for schizophrenia. In schizophrenia patients, it is possible that the $M A G$ rs720308 AA genotype could predict risk for poorer outcome or decreased cognitive reserve based on its effects on left temporal lobe volume. At the same time, effects of the MAG gene on left temporal volume may help explain heterogeneity of findings in neuroimaging studies of the temporal lobe in schizophrenia.

We found that $M A G$ genotype interacted with diagnosis to predict gray matter volume in the parietal lobe. Dysfunction of the parietal lobe may play a critical role in schizophrenia pathophysiology, as it is a key functional domain for many cognitive processes commonly affected in schizophrenia (Torrey, 2007). Compared to the temporal lobe, far fewer reports of parietal lobe volume postmortem or in vivo with MRI have been published in schizophrenia. Those examining parietal tissue have focused on Brodmann area 7 and found dysregulation of several genes involved in apoptotic pathways and nerve maturation, but not of MAG itself (Katsel et al., 2005; Haroutunian et al., 2007). Nevertheless, our finding here provides an intriguing potential role for $M A G$ gene variation in parietal lobe volume in schizophrenia. Similar to the heterogeneity in temporal lobe findings, $M A G$ gene variation may provide one explanation for heterogeneity of parietal lobe findings in schizophrenia. A challenge in studying the parietal lobe is its anatomical variability, making it a difficult region to parcellate effectively. Furthermore, the histological division of inferior parietal structures, namely the angular and supramarginal gyri (Brodmann areas 39 and 40 , respectively), has been reevaluated and seven distinct cytoarchitectonic areas identified using an observer-independent method (Caspers et al., 2006). With the advent of superior neuroanatomical parcelation methods in neuroimaging, future work might be able to examine the influence of $M A G$ genotype in these more specialized regions.

Several potential biological mechanisms may explain the observed effects of $M A G$ gene variation on brain morphology. First, variation in the $M A G$ gene could potentially be responsible for the lower levels of MAG observed in postmortem schizophrenia brain. Second, regional differences of $M A G$ expression may be responsible for myelin abnormalities that have been implicated in a number of demyelinating diseases bearing symptomatic congruency to schizophrenia (Davis et al., 2003). Myelin abnormalities have also been shown to result in axonal degeneration and a reduction in connectivity (Stewart and Davis, 2004), key elements of the myelin-oligodendrocyte hypothesis of schizophrenia pathology. Third, the effects of $M A G$ on gray matter may occur indirectly. The MAG knockout mouse shows reduced density of pyramidal cell basal dendrites of the prefrontal cortex (Segal et al., 2007). Lack of MAG expression, and subsequent effects on other myelin system genes, could affect the ability of satellite oligodendrocytes (in gray matter) to support surrounding neurons (Baumann and Pham-Dinh, 2001). Finally, genetic variation in MAG may influence axonal organization and sprouting since MAG acts as part of a myelin-mediated complex that functions to inhibit axonal growth (Voineskos, 2009). This complex consists of several proteins encoded for by myelin related genes, including the $M A G$ gene, the myelin-oligodendrocyte glycoprotein $(M O G)$ gene, and the receptor tyrosine kinase erbB3 (ErbB3) gene, which bind to the Nogo-66 receptor (gene located in the 22q11 chromosomal region; Budel et al., 2008). Recent data supports that this complex may play a role in the etiopathogenesis of schizophrenia (Budel et al., 2008).

The MAG gene transcript is known to undergo alternative splicing and it is possible that the intronic SNPs investigated here may play a role in this process, though molecular studies will be needed to identify the functional effect of variation at these loci. An exploratory in silico analysis [using the online TFSEARCH search tool (Akiyama; Heinemeyer et al., 1998)] of potential transcription factor (TF) binding sites at these loci has found a decrease in 
binding potential of the sex-determining region $\mathrm{Y}(S R Y)$ protein predicted by the rs720309 $\mathrm{T}$ allele (corresponding to the rs720308 A allele). Interestingly, this male-specific TF is highly expressed in the brain and involved in the biochemical regulation of dopaminergic neurons of the nigrostriatal system (Dewing et al., 2006). Although this raises the possibility of sex interactions, we have an insufficient sample size to test such a hypothesis here. Additionally, it should be noted that these SNPs are not near the promoter region of the MAG gene - leaving the possibility of distal enhancer effects of this TF on MAG expression.

There are several limitations that should be considered in this study. First, as in any structural neuroimaging investigation, partial volume effects can lead to misclassification of tissue. Second, while it is possible that our association is a false positive result, the fact that the present finding is in the same direction as our previous $M A G$ imaging-genetics result on total cortical gray matter volume reduces this concern. Third, newer imaging techniques for evaluating white matter may reveal associations with $M A G$ gene variants. We are addressing this as part of our ongoing studies. Finally, medication effects on brain volumes cannot be ruled out, despite the fact that no relationship with chlorpromazine equivalent dose was found. Our previous finding of association of this

\section{REFERENCES}

Ad-Dab'bagh, Y., Einarson, D., Lyttelton, O., Muehlboeck, J. S., Mok, K., Ivanov, O., Vincent, R. D., Lepage, C., Lerch, J., Fombonne, E., and Evans, A. C. (2006). "The CIVET image-processing environment: a fully automated comprehensive pipeline for anatomical neuroimaging research," in Poster Presentation at the 12th Annual Meeting of the Organization for Human Brain Mapping (OHBM), Florence.

Aston, C., Jiang, L., and Sokolov, B. P. (2004). Microarray analysis of postmortem temporal cortex from patients with schizophrenia. J. Neurosci. Res. 77, 858-866.

Baumann, N., and Pham-Dinh, D. (2001). Biology of oligodendrocyte and myelin in the mammalian central nervous system. Physiol. Rev. 81, 871-927.

Budel, S., Padukkavidana, T., Liu, B. P., Feng, Z., Hu, F., Johnson, S., Lauren, J., Park, J. H., McGee, A. W., Liao, J., Stillman, A., Kim, J.E., Yang, B.-Z., Sodi, S., Gelernter, J., Zhao, H., Hisama, F., Arnsten, A. F. T., and Strittmatter, S. M. (2008). Genetic variants of Nogo-66 receptor with possible association to schizophrenia block myelin inhibition of axon growth. J. Neurosci. 28, 13161-13172.

Caspers, S., Geyer, S., Schleicher, A., Mohlberg, H., Amunts, K., and Zilles, K. (2006). The human inferior parietal cortex: cytoarchitectonic parcellation and interindividual variability. Neuroimage 33, 430-448.

Collins, D. (1994). The crime of failing to record instructions. N. Z. Med. J. 107, 40-41.

Collins, D., Holmes, C., Peters, T., and Evans, A. (1995). Automatic 3D model-based neuroanatomical segmentation. Hum. Brain Mapp. 3, 190-208.

Davis, K. L., Stewart, D. G., Friedman, J. I., Buchsbaum, M., Harvey, P. D., Hof, P. R., Buxbaum, J., and Haroutunian, V. (2003). White matter changes in schizophrenia: evidence for myelin-related dysfunction. Arch. Gen. Psychiatry 60, 443-456.

Dewing, P., Chiang, C. W. K., Sinchak, K., Sim, H., Fernagut, P. O., Kelly, S., Chesselet, M. F., Micevych, P. E., Albrecht, K. H., Harley, V. R., and Vilain, E. (2006). Direct regulation of adult brain function by the malespecific factor SRY. Curr. Biol. 16, 415-420.

Dracheva, S., Davis, K. L., Chin, B., Woo, D. A., Schmeidler, J., and Haroutunian, V. (2006). Myelin-associated mRNA and protein expression deficits in the anterior cingulate cortex and hippocampus in elderly schizophrenia patients. Neurobiol. Dis. 21, 531-540.

Fannon, D., Chitnis, X., Doku, V., Tennakoon, L., O'Ceallaigh, S., Soni, W., Sumich, A., Lowe, J., Santamaria, M., and Sharma, T. (2000). Features of structural brain abnormality variant with cortical gray matter volume in a largely unmedicated, first episode schizophrenia sample (Voineskos et al., 2008) should also help alleviate concerns regarding this potential confound.

In conclusion, we have identified that variation in the MAG gene influences temporal gray matter volumes in healthy controls and chronic schizophrenia patients, and parietal gray matter volumes in schizophrenia patients only. Our findings highlight a potential risk mechanism for temporal lobe volume reduction in schizophrenia via variation at the MAG gene, and that heterogeneity in structural neuroimaging findings in schizophrenia may be in part due to genetic variation in a given sample. Furthermore, the $M A G$ gene variants may help parse out molecular subtypes of schizophrenia that could have important implications for treatment decisions in the future. Overall, our findings highlight the importance of $M A G$ gene variation in brain morphometry in schizophrenia.

\section{ACKNOWLEDGMENTS}

Centre for Addiction and Mental Health (CAMH), CAMH Foundation, Canadian Institutes of Health Research (CIHR), National Alliance for Research on Schizophrenia and Depression (NARSAD), University of Toronto. detected in first-episode psychosis. Am. J. Psychiatry 157, 1829-1834.

First, M. B. Sr., Gibbon, M., and Williams, J. B. W. (1995). Structured Clinical Interview for DSMIV Axis I Disorders, Patient Edition (SCID-P). New York, NY: Biometrics Research.

Fornito, A., Yücel, M., Patti, J., Wood, S. J., and Pantelis, C. (2009). Mapping grey matter reductions in schizophrenia: an anatomical likelihood estimation analysis of voxelbased morphometry studies. Schizophr. Res. 108, 104-113.

Hakak, Y., Walker, J. R., Li, C., Wong, W. H., Davis, K. L., Buxbaum, J. D., Haroutunian, V., and Fienberg, A. A. (2001). Genome-wide expression analysis reveals dysregulation of myelination-related genes in chronic schizophrenia. Proc. Natl. Acad. Sci. U.S.A. 98, 4746-4751.

Haroutunian, V., Katsel, P., Dracheva, S., Stewart, D. G., and Davis, K. L. (2007). Variations in oligodendrocyte-related gene expression across multiple cortical regions: implications for the pathophysiology of schizophrenia. Int. J. Neuropsychopharmacol. 10, 565-573.

Heinemeyer, T., Wingender, E., Reuter I., Hermjakob, H., Kel, A., Kel, O., Ignatieva, E., Ananko, E., Podkolodnaya, O., Kolpakov, F., Podkolodny N., and Kolchanov, N. (1998). Databases on transcriptional regulation: TRANSFAC, TRRD, and COMPEL. Nucleic Acids Res. 26, 364-370.
Jenkinson, M., Bannister, P. R., Brady, J. M., and Smith, S. M. (2002). Improved optimisation for the robust and accurate linear registration and motion correction of brain images. Neuroimage 17, 825-841.

Jenkinson, M., and Smith, S. M. (2001). A global optimisation method for robust affine registration of brain images. Med. Image Anal. 5, 143-156.

Katsel, P., Davis, K. L., and Haroutunian, V. (2005). Variations in myelin and oligodendrocyte-related gene expression across multiple brain regions in schizophrenia: a gene ontology study. Schizophr. Res. 79, 157-173.

Lahiri, D. K., and Nurnberger, J. Jr. (1991). A rapid non-enzymatic method for the preparation of HMW DNA from blood for RFLP studies. Nucleic Acids Res. $19,5444$.

Mazziotta, J., Toga, A., Evans, A., Fox, P., Lancaster, J., Zilles, K., Woods R., Paus, T., Simpson, G., Pike, B., Holmes, C., Collins, L., Thompson, P., MacDonald, D., Iacoboni, M., Schormann, T., Amunts, K., Palomero-Gallagher, N., Geyer, S., Parsons, L., Narr, K., Kabani, N., Le Goualher, G., Feidler, J., Smith, K., Boomsma, D., Hulshoff Pol, H., Cannon, T., Kawashima, R., and Mazoyer, B. (2001). A fourdimensional probabilistic atlas of the human brain. J. Am. Med. Inform. Assoc. 8, 401-430. 
Nguyen, T., Mehta, N. R., Conant, K., Kim, K.-J., Jones, M., Calabresi, P. A., Melli, G., Hoke, A., Schnaar, R. L., Ming, G.-L., Song, H., Keswani, S. C., and Griffin, J. W. (2009). Axonal protective effects of the myelinassociated glycoprotein. J. Neurosci. 29, 630-637.

Oldfield, R. C. (1971). The assessment and analysis of handedness: the Edinburgh inventory. Neuropsychologia 9, 97-113.

Patenaude, B. (2007). Bayesian Statistical Models of Shape and Appearance for Subcortical Brain Segmentation. Ph.D. thesis, University of Oxford, Oxford.

Patenaude, B., Smith, S. M., Kennedy, D., and Jenkinson, M. (2011). A Bayesian model of shape and appearance for subcortical brain. Neuroimage 56, 907-922.

Ross, C. A., and Pearlson, G. D. (1996). Schizophrenia, the heteromodal association neocortex and development: potential for a neurogenetic approach. Trends Neurosci. 19, 171-176.

Schlaepfer, T. E., Harris, G. J., Tien, A. Y., Peng, L. W., Lee, S., Federman, E. B., Chase, G. A., Barta, P. E., and Pearlson, G. D. (1994). Decreased regional cortical gray matter volume in schizophrenia. Am. J. Psychiatry 151, 842-848.

Segal, D., Koschnick, J. R., Slegers, L. H. A., and Hof, P. R. (2007). Oligodendrocyte pathophysiology: a new view of schizophrenia. Int. J. Neuropsychopharmacol. 10, 503-511.
Shenton, M. E., Whitford, T. J., and Kubicki, M. (2010). Structural neuroimaging in schizophrenia: from methods to insights to treatments. Dialogues Clin. Neurosci. 12, 317-332.

Sled, J. G., Zijdenbos, A. P., and Evans, A. C. (1998). A nonparametric method for automatic correction of intensity nonuniformity in MRI data. IEEE Trans. Med. Imaging 17, 87-97.

Smith, S. M. (2002). Fast robust automated brain extraction. Hum. Brain Mapp. 17, 143-155.

Smith, S. M., De Stefano, N., Jenkinson, M., and Matthews, P. M. (2001). Normalised accurate measurement of longitudinal brain change. J. Comput. Assist. Tomogr. 25, 466-475.

Smith, S. M., Zhang, Y., Jenkinson, M., Chen, J., Matthews, P. M., Federico, A., and De Stefano, N. (2002). Accurate, robust, and automated longitudinal and cross-sectional brain change analysis. Neuroimage 17, 479-489.

Stewart, D. G., and Davis, K. L. (2004). Possible contributions of myelin and oligodendrocyte dysfunction to schizophrenia. Int. Rev. Neurobiol. 59, 381-424.

Sun, J., Maller, J. J., Guo, L., and Fitzgerald, P. B. (2009). Superior temporal gyrus volume change in schizophrenia: a review on region of interest volumetric studies. Brain Res. Rev. 61, 14-32.

Tohka, J., Zijdenbos, A., and Evans, A. (2004). Fast and robust parameter estimation for statistical partial volume models in brain MRI. Neuroimage 23, 84-97.

Torrey, E. F. (2007). Schizophrenia and the inferior parietal lobule. Schizophr. Res. 97, 215-225.

Voineskos, A. N. (2009). Converging evidence for the Nogo-66 receptor gene in schizophrenia. J. Neurosci. 29, 5045-5047.

Voineskos, A. N., Lang, D. J., Zai, G., Bulgin, N., Shaikh, S., Su, W., Kopala, L. C., MacEwan, G. W., Thornton, A. E., Smith, G. N., Austin, J. C., Honer, W. G., and Kennedy, J. L. (2008). MAG gene variation and cortical gray matter volume in first episode schizophrenia. Brain Imaging Behav. 2, 117-122.

Wan, C., Yang, Y., Feng, G., Gu, N., Liu, H., Zhu, S., He, L. and Wang, L. (2005). Polymorphisms of myelin-associated glycoprotein gene are associated with schizophrenia in the Chinese Han population. Neurosci. Lett. 388 126-131.

Yang, Y. F., Qin, W., Shugart, Y. Y., He, G., Liu, X. M., Zhou, J., Zhao, X. Z., Chen, Q., La, Y. J., Xu, Y. F., Li, X. W., Gu, N. F., Feng, G. Y., Song, H., Wang, P., and He, L. (2005). Possible association of the MAG locus with schizophrenia in a Chinese Han cohort of family trios. Schizophr. Res. 75, 11-19.

Zhang, Y., Brady, M., and Smith, S. (2001). Segmentation of brain MR images through a hidden Markov random field model and the expectation maximization algorithm. IEEE Trans. Med. Imaging 20, 45-57.

Zijdenbos, A. P., Forghani, R., and Evans, A. C. (2002). Automatic "pipeline" analysis of 3-D MRI data for clinical trials. IEEE Trans. Med. Imaging 21, 1280-1291.

Conflict of Interest Statement: The authors declare that the research was conducted in the absence of any commercial or financial relationships that could be construed as a potential conflict of interest.

Received: 27 February 2012; accepted: 14 April 2012; published online: 03 May 2012.

Citation: Felsky D, Voineskos AN, Lerch JP, Nazeri A, Shaikh SA, Rajji TK, Mulsant BH and Kennedy JL (2012) Myelin-associated glycoprotein gene and brain morphometry in schizophrenia. Front. Psychiatry 3:40. doi: 10.3389/fpsyt.2012.00040

This article was submitted to Frontiers in Neuropsychiatric Imaging and Stimulation, a specialty of Frontiers in Psychiatry. Copyright (C) 2012 Felsky, Voineskos, Lerch, Nazeri, Shaikh, Rajji, Mulsant and Kennedy. This is an open-access article distributed under the terms of the Creative Commons Attribution Non Commercial License, which permits noncommercial use, distribution, and reproduction in other forums, provided the original authors and source are credited. 